TRANSACTIONS OF THE

AMERICAN MATHEMATICAL SOCIETY

Volume 365, Number 12, December 2013, Pages 6199-6216

S 0002-9947(2013)05741-8

Article electronically published on March 25, 2013

\title{
THE CONSTRUCTIBLE TOPOLOGY ON SPACES OF VALUATION DOMAINS
}

\author{
CARMELO A. FINOCCHIARO, MARCO FONTANA, AND K. ALAN LOPER
}

\begin{abstract}
We consider properties and applications of a compact, Hausdorff topology called the "ultrafilter topology" defined on an arbitrary spectral space and we observe that this topology coincides with the constructible topology. If $K$ is a field and $A$ a subring of $K$, we show that the space $\operatorname{Zar}(K \mid A)$ of all valuation domains, having $K$ as the quotient field and containing $A$, (endowed with the Zariski topology) is a spectral space by giving in this general setting the explicit construction of a ring whose Zariski spectrum is homeomorphic to $\operatorname{Zar}(K \mid A)$. We extend results regarding spectral topologies on the spaces of all valuation domains and apply the theory developed to study representations of integrally closed domains as intersections of valuation overrings. As a very particular case, we prove that two collections of valuation domains of $K$ with the same ultrafilter closure represent, as an intersection, the same integrally closed domain.
\end{abstract}

\section{INTRODUCTION}

The motivations for studying spaces of valuation domains come from various directions and, historically, mainly from Zariski's work for building up algebraic geometry by algebraic means (see [30] and [32]), from rigid algebraic geometry started by J. Tate (see [29], 14, and [20]) and from real algebraic geometry (see [27] and [20]). For a deeper insight on this topic, see the paper by Huber-Knebusch [21].

Let $K$ be a field and let $A$ be a subring of $K$. The goal of this paper is to extend results in the literature concerning topologies on the collection of valuation domains which have $K$ as the quotient field and $A$ as a subring, and to provide some applications of these results to the representations of integrally closed domains as intersections of valuation overrings. We denote this collection by $\operatorname{Zar}(K \mid A)$. In the case where $A$ is the prime subring of $K$, then $\operatorname{Zar}(K \mid A)$ includes all valuation domains with $K$ as the quotient field and we denote it simply by $\operatorname{Zar}(K)$. A first topological approach to the space $\operatorname{Zar}(K)$ is due to Zariski who proved the quasicompactness of this space, endowed with what is now called the Zariski topology (see 31] and 32]). It was later proven, and rediscovered by several authors with a variety of different techniques, that if $K$ is the quotient field of $A$, then $\operatorname{Zar}(K \mid A)$ endowed with Zariski's topology is a spectral space in the sense of Hochster [17] (see [3], 4, 21] and the appendix of [22]).

Received by the editors October 7, 2010 and, in revised form, March 29, 2011 and August 11, 2011.

2010 Mathematics Subject Classification. Primary 13A18, 13F05, 13 G05.

During the preparation of this paper, the first two authors were partially supported by a research grant PRIN-MiUR. 
In Section 2, we start by recalling the definition and the basic properties of the constructible topology on an arbitrary topological space, using the notation introduced in [28, Section 2] (for further information cf. [2, §4], [16, (I.7.2.11) and (I.7.2.12)], [17]). Then, we provide a description of the closure in the constructible topology of any subset of a spectral space by using ultrafilters and "ultrafilter limit points" (definition to be given later). As an application, we obtain a new proof that the ultrafilter topology on the prime spectrum of a commutative ring $R$, introduced in [13, is identical to the classical constructible topology on this space.

Section 3 is devoted to the study of the space $\operatorname{Zar}(K \mid A)$ for any subring $A$ of $K$, endowed with the Zariski topology or the constructible topology. The versatility of the ultrafilter approach to the constructible topology is demonstrated in this section and in Section 4, where we make use of Kronecker function rings. The key result in Section 3 is a proof that the space $\operatorname{Zar}(K \mid A)$ is spectral with respect to the constructible (and to the Zariski) topology by giving, in this general setting, the explicit construction of a ring whose prime spectrum is canonically homeomorphic to $\operatorname{Zar}(K \mid A)$. This is broader than the results of Dobbs, Fedder, and Fontana (cf. [3] and [4]), who proved their results in the case where $K$ is the quotient field of $A$ (and only considering the case of the Zariski topology).

Especially noteworthy in Section 4 are the applications of the topological properties of $\operatorname{Zar}(K \mid A)$, endowed with the constructible topology (or, with the inverse topology, in the sense of Hochster [17]), to the representations of integrally closed domains as intersections of valuation overrings. For example, Proposition 4.1 indicates that two collections of valuation domains with the same constructible closure will represent the same domain. Similarly, Corollary 4.14 indicates how the constructible topological structure of a collection of valuation domains determines the associated finite-type e.a.b. semistar operation. We also apply these results to the class of vacant domains (those domains which have a unique Kronecker function ring). In particular, Corollaries 4.10 and 4.11 use the constructible topology to characterize vacant domains. We then relate closure in the inverse topology to closure in the constructible topology and restate our results concerning e.a.b. semistar operations in terms of the inverse topology. For some distinguished classes of domains, other important contributions on this circle of ideas were given in [23, 24], [25], and [26].

\section{Preliminaries, SPeCtral spaCes and Ultrafilter limit Points}

If $X$ is a set, we denote by $\mathscr{B}(X)$ the collection of all subsets of $X$, and by $\mathscr{B}_{\text {fin }}(X)$ the collection of all finite subsets of $X$. Moreover, if $\mathscr{G}$ is a nonempty subset of $\mathscr{B}(X)$, then we will simply denote by $\cap \mathscr{G}$ (resp. $\bigcup \mathscr{G}$ ) the set obtained by intersection (resp. union) of all the subsets of $X$ belonging to $\mathscr{G}$, i.e., $\bigcap^{\mathscr{G}}:=$ $\bigcap\{G \mid G \in \mathscr{G}\}$ (resp. $\bigcup \mathscr{G}:=\bigcup\{G \mid G \in \mathscr{G}\}$ ).

Recall that a nonempty collection $\mathscr{F}$ of subsets of $X$ is said to be a fiter on $X$ if the following conditions are satisfied: (a) $\emptyset \notin \mathscr{F}$; (b) if $F, G \in \mathscr{F}$, then $F \cap G \in \mathscr{F}$; (c) if $F, G \in \mathscr{B}(X), F \subseteq G$, and $F \in \mathscr{F}$, then $G \in \mathscr{F}$.

Let $\mathcal{F}(X)$ be the set of all filters on $X$, partially ordered by inclusion. We say that a filter $\mathscr{F}$ on $X$ is an ultrafilter on $X$ if it is a maximal element in $\mathcal{F}(X)$. In the following, we will denote the collection of all ultrafilters on a set $X$ by $\boldsymbol{\beta}(X)$. 
For each $x \in X$, it is immediately seen that $\beta_{X}^{x}:=\beta^{x}:=\{Z \in \mathscr{B}(X) \mid x \in Z\}$ is an ultrafilter on $X$, called the trivial (or fixed or principal) ultrafilter of $X$ centered on $x$.

Recall that a spectral space is a topological space homeomorphic to the prime spectrum of a ring, equipped with the Zariski topology. The spectral spaces were characterized by Hochster in 1969 as quasi-compact Kolmogoroff topological spaces, with a quasi-compact open basis stable under finite intersections and such that every nonempty irreducible closed subspace has a generic point [17, Proposition 4].

Let $\mathcal{X}$ be a topological space. With the notation used in [28, Section 2] we set

$$
\begin{aligned}
\stackrel{\circ}{\mathcal{K}}:=\mathcal{K}(\mathcal{X}):= & \{U \mid U \subseteq \mathcal{X}, U \text { open and quasi-compact in } \mathcal{X}\}, \\
\overline{\mathcal{K}}:=\overline{\mathcal{K}}(\mathcal{X}):= & \{\mathcal{X} \backslash U \mid U \in \mathcal{K}(\mathcal{X})\}, \\
\mathcal{K}:=\mathcal{K}(\mathcal{X}):= & \text { the Boolean algebra of the subsets of } \mathcal{X} \text { generated } \\
& \text { by } \mathcal{K}(\mathcal{X}),
\end{aligned}
$$

i.e., $\mathcal{K}(\mathcal{X})$ is the smallest subset of $\mathscr{B}(\mathcal{X})$ containing $\mathcal{K}(\mathcal{X})$ and closed with respect to finite $\cup, \cap$, and complementation. As in [28, we call the constructible topology on $\mathcal{X}$ the topology on $\mathcal{X}$ having $\mathcal{K}(\mathcal{X})$ as a basis (for the open sets). We denote by $\mathcal{X}^{\text {cons }}$ the set $\mathcal{X}$ equipped with the constructible topology and we call constructible sets of $\mathcal{X}$ the elements of $\mathcal{K}(\mathcal{X})$ (for Noetherian topological spaces, this notion coincides with that given in $[2, \S 4])$ and proconstructible sets the closed sets of $\mathcal{X}^{\text {cons }}$.

Now consider $\mathcal{Y}$ a subset of $\mathcal{X}$. In the following, we denote by $\operatorname{Cl}(\mathcal{Y})$ (respectively, $C l^{\text {cons }}(\mathcal{Y})$ ) the closure of $\mathcal{Y}$, with respect to the given topology (respectively, the constructible topology) on $\mathcal{X}$.

Assume that $\mathcal{X}$ is a spectral space. In this case, the set $\mathcal{K}(:=\mathcal{K}(\mathcal{X}))$ is a basis of the topology on $\mathcal{X}$ and it is closed under finite intersections. The constructible topology on $\mathcal{X}$ is the coarsest topology for which $\mathcal{K}$ is a collection of clopen sets and $\mathcal{X}^{\text {cons }}$ is a compact, Hausdorff topological space.

We can consider on $\mathcal{X}$ the usual partial order, defined by

$$
x \preccurlyeq y: \Longleftrightarrow y \in C l(\{x\}) .
$$

If $\mathcal{Y}$ is a subset of $\mathcal{X}$, set

$\mathcal{Y}^{\text {sp }}:=\{x \in \mathcal{X} \mid y \preccurlyeq x$, for some $y \in \mathcal{Y}\}, \mathcal{Y}^{\text {gen }}:=\{x \in \mathcal{X} \mid x \preccurlyeq y$, for some $y \in \mathcal{Y}\}$.

Then $\mathcal{Y}^{\text {sp }}$ (respectively, $\mathcal{Y}^{\text {gen }}$ ) is the closure under specializations (respectively, the closure under generizations or the generic closure) of $\mathcal{Y}$.

Following [17, we can also endow the spectral space $\mathcal{X}$ with the so-called inverse topology (or dual topology), that is, the topology whose basis of closed sets is the set $\mathcal{K}(\mathcal{X})$ of all open and quasi-compact subspaces of $\mathcal{X}$ (with respect to the given spectral topology). We denote by $\mathcal{X}^{\text {inv }}$ the set $\mathcal{X}$, endowed with the inverse topology. By [17, Proposition 8], $\mathcal{X}^{\text {inv }}$ is a spectral space and its constructible topology is clearly equal to the constructible topology associated to the given spectral topology on $\mathcal{X}$. The following fact provides a motivation for the name given to this topology.

2.1. Proposition ([17, Proposition 8]). Let $\mathcal{X}$ be a spectral space. Denote by (respectively, $\preccurlyeq^{\prime}$ ) the order induced by the given spectral topology (respectively, the inverse topology) on $\mathcal{X}$. Then, for any $x, y \in \mathcal{X}$, we have

$$
\left.x \preccurlyeq y(\text { i.e., } y \in C l(\{x\})) \Leftrightarrow y \preccurlyeq^{\prime} x \text { (i.e., } x \in C l^{i n v}(\{y\})\right) \text {. }
$$


2.2. Remark. Let $\mathcal{X}$ be a spectral space and $\mathcal{Y}$ be a subset of $\mathcal{X}$. Then, by 8 , Lemma 1.1], [17, Corollary to Theorem 1] and Proposition 2.1 we have

$$
C l(\mathcal{Y})=\left(C l^{\text {cons }}(\mathcal{Y})\right)^{\text {sp }} \text { and } \quad C l^{\text {inv }}(\mathcal{Y})=\left(C l^{\text {cons }}(\mathcal{Y})\right)^{\text {gen }} \text {. }
$$

2.3. Proposition. Let $\mathcal{X}$ be a spectral space, $\mathcal{Y}$ be a subset of $\mathcal{X}$ and $\mathscr{U}$ be an ultrafilter on $\mathcal{Y}$. Set

$$
\begin{aligned}
\overline{\mathcal{K}}_{\mathcal{Y}, \mathscr{U}}:=\{\mathcal{X} \backslash U \mid U \in \mathcal{K} \text { and } \mathcal{Y} \backslash U \in \mathscr{U}\} \\
=\{C \in \overline{\mathcal{K}} \mid C \cap \mathcal{Y} \in \mathscr{U}\} \\
\mathcal{K}_{\mathcal{Y}, \mathscr{U}}:=\overline{\mathcal{K}}_{\mathcal{Y}, \mathscr{U}} \bigcup\{U \in \mathcal{K} \mid U \cap \mathcal{Y} \in \mathscr{U}\} .
\end{aligned}
$$

Then, the following statements hold.

(1) The set $\bigcap \mathcal{K}_{\mathcal{Y}, \mathscr{U}}$ is a singleton, the set $\bar{K}_{\mathcal{Y}, \mathscr{U}}:=\bigcap \overline{\mathcal{K}}_{\mathcal{Y}, \mathscr{U}}$ is an irreducible closed subset of $\mathcal{X}$. The generic point of $\bar{K}_{\mathcal{Y}, \mathscr{U}}$ is the unique point $x_{\mathscr{U}}:=$ $x_{\mathcal{Y}, \mathscr{U}} \in \bigcap \mathcal{K}_{\mathcal{Y}, \mathscr{U}}$. We will call the point $x_{\mathscr{U}} \in \mathcal{X}$ the ultrafilter limit point of $\mathcal{Y}$, with respect to $\mathscr{U}$.

(2) $C l^{\text {cons }}(\mathcal{Y})=\{x \mathcal{Y}, \mathscr{U} \mid \mathscr{U} \in \boldsymbol{\beta}(\mathcal{Y})\}$.

Proof. (1) By construction $\mathcal{K}_{\mathcal{Y}, \mathscr{U}}$ is a collection of closed subsets of $\mathcal{X}^{\text {cons }}$ with the finite intersection property. Thus, $\cap \mathcal{K}_{\mathcal{Y}, \mathscr{U}}$ is nonempty. Since $\mathcal{X}$ is, in particular, a topological space satisfying $\mathrm{T}_{0}$ axiom, the conclusion will follow if we show that if $x \in \bigcap \mathcal{K}_{\mathcal{Y}, \mathscr{U}}$, then $\bar{K}_{\mathcal{Y}, \mathscr{U}}=C l(\{x\})$. Since $\overline{\mathcal{K}}_{\mathcal{Y}, \mathscr{U}} \subseteq \mathcal{K}_{\mathcal{Y}, \mathscr{U}}$ and $\bar{K}_{\mathcal{Y}, \mathscr{U}}$ is closed in $\mathcal{X}$, it follows that $C l(\{x\}) \subseteq \bar{K}_{\mathcal{Y}, \mathscr{U}}$.

Conversely, let $z \in \bar{K}_{\mathcal{Y}, \mathscr{U}}$ and let $U$ be an open neighborhood of $z$, with respect to the spectral topology. Without loss of generality, we can assume that $U \in \dot{\mathcal{K}}$. We have $\mathcal{Y} \cap U \in \mathscr{U}$ (otherwise, since $\mathscr{U}$ is an ultrafilter on $\mathcal{Y}, \mathcal{Y} \backslash U \in \mathscr{U}$, so $\mathcal{X} \backslash U \in \overline{\mathcal{K}}_{\mathcal{Y}, \mathscr{U}}$, and thus in particular $z \in \mathcal{X} \backslash U$ : a contradiction), hence $U \in \mathcal{K}_{\mathcal{Y}, \mathscr{U}}$ and $x \in U$, in particular. Then, $z \in C l(\{x\})$.

(2) Let $\mathscr{U}$ be an ultrafilter on $\mathcal{Y}$ and let $\Omega$ be an open neighborhood of $x_{\mathscr{U}}$, with respect to the constructible topology. Since the collection of all clopen sets of $\mathcal{X}$ is a basis for the open sets of $\mathcal{X}^{\text {cons }}$, we can assume, without loss of generality, that $\Omega=U \cap(\mathcal{X} \backslash V)$, for some $U, V \in \mathcal{K}$. It follows immediately that $U \cap \mathcal{Y}$ and $\mathcal{Y} \backslash V$ belong to $\mathscr{U}$ (otherwise, either $\mathcal{X} \backslash U$ or $V$ would belong to $\mathcal{K}_{\mathcal{Y}, \mathscr{U}}$, then we would have a contradiction since $\bigcap \mathcal{K}_{\mathcal{Y}, \mathscr{U}}=\left\{x_{\mathscr{U}}\right\}$ and $x_{\mathscr{U}} \in U \cap(\mathcal{X} \backslash V)$ ). Thus, $\Omega \cap \mathcal{Y} \in \mathscr{U}$ and it is, in particular, nonempty. This proves that $x_{\mathscr{U}} \in C l^{\text {cons }}(\mathcal{Y})$.

Conversely, let $x \in C l^{\text {cons }}(\mathcal{Y})$. Note that the following collection of sets (subsets of $\mathcal{Y})$

$$
\mathcal{G}:=\{\mathcal{Y} \cap U \cap(\mathcal{X} \backslash V) \mid U, V \in \stackrel{\circ}{\mathcal{K}}, x \in U \cap(\mathcal{X} \backslash V)\}
$$

has the finite intersection property, since $\mathcal{K}$ is a collection of clopen sets of the compact space $\mathcal{X}^{\text {cons }}$. Pick an ultrafilter $\mathscr{U}$ on $\mathcal{Y}$ such that $\mathcal{G} \subseteq \mathscr{U}$. We claim that $x=x_{\mathscr{U}}$. To see this, since $\mathcal{X}$ is a $\mathrm{T}_{0}$ topological space, it suffices to show that $x$ and $x_{\mathscr{U}}$ have the same set of open neighborhoods in $\mathcal{X}$ (with respect to the given (spectral) topology). Let $U$ be an open and quasi-compact neighborhood of $x$. It follows $\mathcal{Y} \cap U \in \mathcal{G} \subseteq \mathscr{U}$. Thus $U \in \mathcal{K}_{\mathcal{Y}, \mathscr{U}}$ and, in particular, $x_{\mathscr{U}} \in U$. Conversely, assume, by contradiction, that there is an open and compact neighborhood $U$ of $x_{\mathscr{U}}$ such that $x \notin U$. Then, $\mathcal{Y} \cap(\mathcal{X} \backslash U) \in \mathcal{G} \subseteq \mathscr{U}$. It follows that $\mathcal{X} \backslash U \in \mathcal{K}_{\mathcal{Y}, \mathscr{U}}$ and $x_{\mathscr{U}} \in \mathcal{X} \backslash U$; a contradiction.

We apply the previous result to the prime spectrum of a ring. 
2.4. Corollary. Let $R$ be a ring, $X:=\operatorname{Spec}(R)$ (equipped with the Zariski topology), $Y$ a subset of $X$ and $\mathscr{U}$ an ultrafilter on $Y$. Then,

$$
P_{\mathscr{U}}:=P_{Y, \mathscr{U}}:=\{a \in R \mid V(a) \cap Y \in \mathscr{U}\}
$$

is a prime ideal which coincides with the ultrafilter limit point $x \mathcal{Y}, \mathscr{U}$ of $X$ defined in Proposition 2.3 .

Proof. By an argument similar to that used in [1, Lemma 2.4] (see also [13]) it can be easily shown that $P_{\mathscr{U}}$ is a prime ideal of $R$. Let $H \in \mathcal{K}_{Y, \mathscr{U}}$. Then $H \cap Y \in \mathscr{U}$ and either $H=\bigcup_{i=1}^{n} D\left(f_{i}\right)(\in \dot{\mathcal{K}}(\operatorname{Spec}(R)))$, for some $f_{1}, f_{2}, \ldots, f_{n} \in R$, or $H=$ $V(I)(\in \overline{\mathcal{K}}(\operatorname{Spec}(R)))$, for some finitely generated ideal $I$ of $R$. In the first case, we have $\bigcup_{i=1}^{n} Y \cap D\left(f_{i}\right) \in \mathscr{U}$, thus $D\left(f_{j}\right) \cap Y \in \mathscr{U}$, for some $j, 1 \leq j \leq n$, and so $P_{Y, \mathscr{U}} \in D\left(f_{j}\right) \subseteq H$. In the other case, we have $V(I) \cap Y \in \mathscr{U}$ and, since $I$ is finitely generated. $P_{Y, \mathscr{U}} \in V(I)=H$. Recalling that $\bigcap \mathcal{K}_{Y, \mathscr{U}}$ is a singleton, the conclusion follows immediately.

2.5. Remark. Let $\mathcal{X}$ be a spectral space and $\mathcal{Y}$ be a subset of $\mathcal{X}$. We say that $\mathcal{Y}$ is ultrafilter closed in $\mathcal{X}$ if $x \mathcal{Y}, \mathscr{U} \in \mathcal{Y}$, for any ultrafilter $\mathscr{U}$ on $\mathcal{Y}$. By Proposition 2.3, it follows that the collection of all the subsets of $\mathcal{X}$ that are ultrafilter closed is the family of closed sets for a topology $\mathcal{X}$, that we call the ultrafilter topology of the spectral space $\mathcal{X}$. If we denote by $\mathcal{X}^{\text {ultra }}$ the space $\mathcal{X}$ endowed with the ultrafilter topology, then by Proposition 2.3 we have $\mathcal{X}^{\text {ultra }}=\mathcal{X}^{\text {cons }}$. Therefore, from Proposition 2.3 and Corollary 2.4, when $\mathcal{X}$ is the prime spectrum of a commutative ring, we reobtain as a particular case [13, Theorem 8].

2.6. Proposition. Let $\mathcal{X}$ be a spectral space and $\mathcal{Y}$ be a quasi-compact subspace of $\mathcal{X}$. Then, the generic closure $\mathcal{Y}^{\text {gen }}$ of $\mathcal{Y}$ in $\mathcal{X}$ is closed in $\mathcal{X}^{\text {cons }}$.

Proof. Preserve the notation of Proposition 2.3, and let $\mathscr{U}$ be an ultrafilter on $\mathcal{Y}^{\text {gen }}$. It is sufficient to show that $x_{\mathscr{U}}:=x_{\mathcal{Y}_{\text {gen }}, \mathscr{U}} \in \mathcal{Y}^{\text {gen }}$. If not, for each $y \in \mathcal{Y}$, there is an open and compact open neighborhood $\Omega_{y}$ of $y$ such that $x_{\mathscr{U}} \notin \Omega_{y}$. By compactness, the open cover $\left\{\Omega_{y} \mid y \in \mathcal{Y}\right\}$ of $\mathcal{Y}$ in $\mathcal{X}^{\text {cons }}$ has a finite subcover, say $\left\{\Omega_{y_{i}} \mid i=1,2, \ldots, n\right\}$. It is easily checked that $\mathcal{Y}^{\text {gen }} \subseteq \bigcup_{i=1}^{n} \Omega_{y_{i}}$, i.e., $\mathcal{Y}^{\text {gen }}=$ $\bigcup_{i=1}^{n}\left(\Omega_{y_{i}} \cap \mathcal{Y}^{\text {gen }}\right) \in \mathscr{U}$. Moreover, since $\mathscr{U}$ is an ultrafilter on $\mathcal{Y}^{\text {gen }}, \Omega_{y_{i}} \cap \mathcal{Y}^{\text {gen }} \in \mathscr{U}$, for some for some $i \in\{1,2, \ldots, n\}$. Thus, by Proposition 2.3(1), we have $x_{\mathscr{U}} \in \Omega_{y_{i}}$; a contradiction.

\section{The Kronecker function Ring (After Halter-Koch) And THE ZARISKI-RIEMANN SURFACE}

Let $K$ be a field and let $A$ be any subring of $K$. Denote by $\operatorname{Zar}(K \mid A)$ the set of all the valuation domains having $K$ as the quotient field and containing $A$ as a subring.

As is well known, Zariski [31] (or, [32, Volume II, Chapter VI, §1, page 110]) introduced and studied the set $Z:=\operatorname{Zar}(K \mid A)$ together with a topological structure defined by taking, as a basis for the open sets, the subsets $B_{F}^{Z}:=\{V \in Z \mid V \supseteq F\}$, for $F$ varying in $\mathscr{B}_{\text {fin }}(K)$, i.e., if $F:=\left\{x_{1}, x_{2}, \ldots, x_{n}\right\}$, with $x_{i} \in K$, then

$$
B_{F}^{Z}=\operatorname{Zar}\left(K \mid A\left[x_{1}, x_{2}, \ldots, x_{n}\right]\right) .
$$

This topology is called the Zariski topology on $Z=\operatorname{Zar}(K \mid A)$ and $Z$, equipped with this topology, denoted also later by $Z^{\mathrm{zar}}$, is usually called the (abstract) ZariskiRiemann surface of $K$ over $A$. 
On the set $Z=\operatorname{Zar}(K \mid A)$ we can also consider the constructible topology, as defined in the previous section, and we denote, as usual, $Z^{\text {cons }}$ the space $Z$ endowed with the constructible topology.

In this section, we show that both $\operatorname{Zar}(K \mid A)^{\text {zar }}$ and $\operatorname{Zar}(K \mid A)^{\text {cons }}$ are spectral spaces, by giving in this general setting the explicit construction of a ring whose prime spectrum, equipped with the Zariski topology (respectively, constructible topology), is homeomorphic to $\operatorname{Zar}(K \mid A)^{\mathrm{zar}}$ (respectively, $\operatorname{Zar}(K \mid A)^{\text {cons }}$ ).

Let $K$ be a field and $T$ an indeterminate over $K$. For every $W \in \operatorname{Zar}(K(T))$, it is well known that $V:=W \cap K \in \operatorname{Zar}(K)$ [15, Theorem 19.16(a)] and conversely, for each $V \in \operatorname{Zar}(K)$, there are infinitely many valuation domains $W$ of $K(T)$ such that $W \cap K=V$, called extensions of $V$ to $K(T)$ [15, Proposition 20.11]. Among the extensions of a valuation $V$ of $K$ to $K(T)$, there is a distinguished one, called the trivial extension of $V$ to $K(T)$, which is $V(T):=V[T]_{M[T]}$, where $M$ is the maximal ideal of $V$ [15, Proposition 18.7].

3.1. Proposition. Let $K$ be a field and $T$ an indeterminate over $K$.

(1) The canonical map $\varphi: \operatorname{Zar}(K(T))^{z a r} \rightarrow \operatorname{Zar}(K)^{z a r}, W \mapsto W \cap K$, is a continuous surjection.

(2) Let $\operatorname{Zar}_{0}(K(T)):=\{V(T) \in \operatorname{Zar}(K(T)) \mid V \in \operatorname{Zar}(K)\}$. Then,

$$
\left.\varphi\right|_{\operatorname{Zar}_{0}(K(T))}: \operatorname{Zar}_{0}(K(T))^{z a r} \rightarrow \operatorname{Zar}(K)^{z a r}
$$

is a homeomorphism.

Proof. (1) The map $\varphi$ is clearly surjective by the previous remarks. It is also a continuous map since, for each finite subset $F$ of $K$ and for each basic open set $B_{F}^{\operatorname{Zar}(K)}$ of $\operatorname{Zar}(K)^{\mathrm{zar}}$, it is straightforward to see that $\varphi^{-1}\left(B_{F}^{\mathrm{Zar}(K)}\right)=B_{F}^{\mathrm{Zar}(K(T))}$.

(2) It is obvious that $\varphi \mid \operatorname{Zar}_{0}(K(T)): \operatorname{Zar}_{0}(K(T))^{\mathrm{zar}} \rightarrow \operatorname{Zar}(K)^{\mathrm{zar}}$ is a bijection and, by (1), is a continuous map. The conclusion will follow if we show that the map $\left.\varphi\right|_{\operatorname{Zar}_{0}(K(T))}$ is also open. Let $h \in K(T) \backslash\{0\}$, say

$$
h:=\frac{a_{0}+a_{1} T+\cdots+a_{r} T^{r}}{b_{0}+b_{1} T+\cdots+b_{s} T^{s}}
$$

with $a_{i}$ and $b_{j}$ in $K$, for $i=0,1, \ldots, r$ and $j=0,1, \ldots, s$. Let $V(T)$ be a valuation domain in $\operatorname{Zar}_{0}(K(T))$, let $v$ be the valuation on $K$ defining $V$ and let $v^{*}$ be the valuation associated to $V(T)$, i.e., $v^{*}(T)=v(1)=0$ and, for each nonzero polynomial $f:=a_{0}+a_{1} T+\cdots+a_{r} T^{r} \in K[T], v^{*}(f):=\inf \left\{v\left(a_{i}\right) \mid i=0,1, \ldots, r\right\}$.

It is easy to see that $V(T) \in B_{h}^{\operatorname{Zar}(K(T))}$ if and only if $v^{*}(h) \geq 0$, that is, if and only if

$$
\inf \left\{v\left(a_{i}\right) \mid i=0,1, \ldots, r\right\} \geq \inf \left\{v\left(b_{j}\right) \mid j=0,1, \ldots, s\right\} .
$$

Now, for all $i \in\{0,1, \ldots, r\}$ and $j \in\{0,1, \ldots, s\}$ such that both $a_{i}$ and $b_{j}$ are nonzero, set

$$
F_{i j}:=\left\{\frac{a_{i}}{b_{j}}, \frac{a_{\lambda}}{a_{i}}, \frac{b_{\mu}}{b_{j}} \mid \lambda=0,1, \ldots, r, \mu=0,1, \ldots, s\right\} .
$$

Then, it is not hard to verify that $\varphi\left(B_{h}^{\operatorname{Zar}_{0}(K(T))}\right)=\bigcup_{i, j} B_{F_{i j}}^{\operatorname{Zar}(K)}$ (see also the proof of [4, Lemma 1]), hence the continuous bijective map $\left.\varphi\right|_{\operatorname{Zar}_{0}(K(T))}$ is open, and so a homeomorphism.

Now recall the following key notion introduced by Halter-Koch [18, Definition 2.1], providing an axiomatic approach to the theory of Kronecker function rings. 
Let $K$ be field, $T$ an indeterminate over $K$, and $R$ a subring of $K(T)$. We call $R$ a $K$-function ring (after Halter-Koch) if $T$ and $T^{-1}$ belong to $R$ and, for each nonzero polynomial $f \in K[T], f(0) \in f(T) R$.

In the next proposition we collect several properties of $K$-function rings that will be useful in the following.

3.2. Proposition. Let $K$ be a field, $T$ an indeterminate over $K$ and let $R$ be a subring of $K(T)$. Assume that $R$ is a $K$-function ring.

(1) If $R^{\prime}$ is a subring of $K(T)$ containing $R$, then $R^{\prime}$ is also a $K$-function ring.

(2) If $\mathscr{R}$ is a nonempty collection of $K$-function rings (in $K(T)$ ), then $\bigcap \mathscr{R}$ is a K-function ring.

(3) $R$ is a Bézout domain with quotient field $K(T)$.

(4) If $f:=f_{0}+f_{1} T+\cdots+f_{r} T^{r} \in K[T]$, then $\left(f_{0}, f_{1}, \ldots, f_{r}\right) R=f R$.

(5) For every valuation domain $V$ of $K, V(T)$ is a $K$-function ring.

Proof. (1), (2), (3) and (4) were proved in [18, Remarks at page 47 and Theorem (2.2)]. To prove (5), observe that if $v$ is the valuation associated to $V$ and $v^{*}$ is the trivial extension of $v$ to $K(T)$ [15, page 218], then $v^{*}(T)=v(1)=0$ or, equivalently, $T$ is invertible in $V(T)$. Moreover, if $f:=f_{0}+f_{1} T+\cdots+f_{r} T^{r} \in K[T]$, then $v^{*}(f) \leq v\left(f_{0}\right)=v^{*}\left(f_{0}\right)$, and so $f(0)=f_{0} \in f V(T)$.

The following fact provides a slight generalization of [19, Theorem 2.3] and its proof is similar to that given by $\mathrm{O}$. Kwegna Heubo, which is based on the work by Halter-Koch [18.

3.3. Proposition. Let $K$ be a field, $T$ an indeterminate over $K$ and $R$ a $K$-function ring. Then, $\operatorname{Zar}(K(T) \mid R)=\operatorname{Zar}_{0}(K(T) \mid R$ ) (i.e., for every valuation domain $W \in$ $\operatorname{Zar}(K(T) \mid R), W=(W \cap K)(T))$.

Proof. Let $W$ be a valuation overring of $R$. First, observe that $V:=W \cap K$ is a valuation ring of $K$ [15, Theorem 19.16(a)]. Now, let $v$ be a valuation of $K$ defining $V$ and let $f:=f_{0}+f_{1} T+\cdots+f_{r} T^{r} \in K[T], f \neq 0$. By Proposition [3.2(1), since $R \subseteq W, W$ is a $K$-function ring. Let $w$ be a valuation of $K(T)$ defining $W$. Since $T$ and $T^{-1}$ belong to $W$, we have $w(T)=0$. Moreover, $\left.w\right|_{K}=v$ and so $w(f) \geq \inf \left\{w\left(f_{i}\right) \mid i=0,1, \ldots, r\right\}=\inf \left\{v\left(f_{i}\right) \mid i=0,1, \ldots, r\right\}$.

On the other hand, by Proposition 3.2(4), $f R=f_{0} R+f_{1}+\cdots+f_{r} R$, and thus $f_{i} \in f R$, for every $i=0,1, \ldots, r$. Since $R \subseteq W$, we have $f_{i} \in f W$ and thus $w\left(f_{i}\right)=v\left(f_{i}\right) \geq w(f)$, for every $i=0,1, \ldots, r$. Therefore, $w(f)=\inf \left\{v\left(f_{i}\right) \mid i=\right.$ $0,1, \ldots, r\}$. This proves that $w=v^{*}$, and hence $W$ is the trivial extension of $V$ in $K(T)$.

3.4. Proposition. Let $K$ be a field, $T$ an indeterminate over $K$, and $R$ a subring of $K(T)$. Then, the following conditions are equivalent:

(i) $R$ is a $K$-function ring.

(ii) $R$ is integrally closed in $K(T)$ and $\operatorname{Zar}(K(T) \mid R)=\operatorname{Zar}_{0}(K(T) \mid R)$.

Proof. (i) $\Rightarrow$ (ii) is already known (Propositions $3.2(3)$ and 3.3 ).

(ii) $\Rightarrow$ (i). Since $R$ is integrally closed in $K(T)$ and $\operatorname{Zar}(K(T) \mid R)=\operatorname{Zar}_{0}(K(T) \mid R)$, then $R=\bigcap \operatorname{Zar}_{0}(K(T) \mid R)$. Now, the conclusion is clear, by Proposition [3.2 $(2)$, (5).

As a consequence of Propositions 3.1 (2) and 3.3, we deduce immediately the following. 
3.5. Corollary. Let $K$ be a field, $T$ an indeterminate over $K$ and $R(\subseteq K(T))$ a $K$-function ring. Set $A_{R}:=R \cap K$. Then, the canonical map $\varphi: \operatorname{Zar}(K(T) \mid R) \longrightarrow$ $\operatorname{Zar}\left(K \mid A_{R}\right), W \mapsto W \cap K$, is a topological embedding, with respect to the Zariski topology.

As an application of the previous corollary we re-obtain in particular [19, Corollary 2.2, Proposition 2.7 and Corollary 2.9]. More precisely, we have the following.

3.6. Corollary. Let $K$ be a field, $A$ any subring of $K$ and $T$ an indeterminate over K. Then,

(1) $\operatorname{Kr}(K \mid A):=\bigcap\{V(T) \mid V \in \operatorname{Zar}(K \mid A)\}$ is a $K$-function ring.

(2) The canonical map $\varphi: \operatorname{Zar}(K(T) \mid \operatorname{Kr}(K \mid A))^{\text {zar }} \rightarrow \operatorname{Zar}(K \mid A)^{z a r}, W \mapsto W \cap$ $K$, is a homeomorphism.

(3) The canonical map $\psi: \operatorname{Spec}(\operatorname{Kr}(K \mid A))^{z a r} \rightarrow \operatorname{Zar}(K \mid A)^{z a r}, Q \mapsto \operatorname{Kr}(K \mid A)_{Q}$ $\cap K$, is a homeomorphism. In particular, $\operatorname{Zar}(K \mid A)^{z a r}$ is a spectral space.

Proof. (1) By Proposition $3.2(2)(5), \operatorname{Kr}(K \mid A)$ is a $K$-function ring (in $K(T)$ ).

(2) Let $R:=\operatorname{Kr}(K \mid A)$, then clearly $R \cap K$ coincides with the integral closure $\bar{A}$ of $A$ in $K$, and therefore $\varphi(\operatorname{Zar}(K(T) \mid R))=\operatorname{Zar}(K \mid A)$. Now (2) follows from Corollary 3.5 .

(3) Recall that, if $\mathcal{A}$ is a Prüfer domain and $\mathcal{K}$ is the quotient field of $\mathcal{A}$, by [4, Proposition 2.2], $\operatorname{Zar}(\mathcal{K} \mid \mathcal{A})^{\mathrm{zar}}$ is canonically homeomorphic to $\operatorname{Spec}(\mathcal{A})^{\mathrm{zar}}$ (under the map $\mathcal{V} \mapsto M_{\mathcal{V}} \cap \mathcal{A}$, where $M_{\mathcal{V}}$ is the maximal ideal of the valuation domain $\mathcal{V}$ of $\mathcal{K}$ containing $\mathcal{A}$ ). Now, the conclusion follows immediately, since $\operatorname{Kr}(K \mid A)$ is a Prüfer domain with quotient field $K(T)$ (Proposition 3.2(3)).

3.7. Remark. Note that the noteworthy progress provided by Corollary 3.6 concerns the case where $A$ is a proper subfield of $K$. As a matter of fact, if $A$ is an integrally closed domain and $K$ is its quotient field, statements (2) and (3) of Corollary 3.6 were already proved in [4, Theorem 2].

By Corollary [3.6 (3), $\operatorname{Zar}(K \mid A)^{\mathrm{zar}}$ is a spectral space. As we will see next, by applying Proposition 2.3 and Remark 2.5. we re-obtain the main statement of 7 , Theorem 3.4].

3.8. Corollary. Let $K$ be a field and $A$ a subring of $K$. If $Y$ is a nonempty subset of $\operatorname{Zar}(K \mid A)$ (equipped with the Zariski topology) and $\mathscr{U}$ is an ultrafilter on $Y$, then

$$
A_{\mathscr{U}}:=A_{Y, \mathscr{U}}:=\left\{x \in K \mid B_{x} \cap Y \in \mathscr{U}\right\}
$$

is the valuation domain of $K$ containing $A$ and it coincides with the ultrafilter limit point $x_{Y, \mathscr{U}}$ of $\operatorname{Zar}(K \mid A)$ defined as in Proposition 2.3 . Moreover, $\operatorname{Zar}(K \mid A)^{\text {cons }}=$ $\operatorname{Zar}(K \mid A)^{u l t r a}$.

Proof. Preserve the notation of Proposition 2.3. By [1, Lemma 2.9] (or [7, Proposition 3.1]), we have $A_{Y, \mathscr{U}} \in \operatorname{Zar}(K \mid A)$. The conclusion will follow by Proposition 2.3 and Remark 2.5] if we show that $A_{Y, \mathscr{U}} \in \bigcap \mathcal{K}_{Y, \mathscr{U}}$. Suppose $H \in \mathcal{K}_{Y, \mathscr{U}}$. By definition, $H \cap Y \in \mathscr{U}$ and either $H=U$ or $H=X \backslash U$, for some open and compact subspace $U$ of $\operatorname{Zar}(K \mid A)^{\text {zar }}$. Since $\mathcal{B}:=\left\{B_{F} \mid F \in \mathscr{B}_{\text {fin }}(K)\right\}$ is a basis of the Zariski topology, $U$ is the union of a finite subfamily $\mathcal{B}^{\prime}$ of $\mathcal{B}$. Thus, if $H=U$ (and so $Y \cap U \in \mathscr{U})$, there exists a set $B_{F} \in \mathcal{B}^{\prime}$ such that $B_{F} \cap Y \in \mathscr{U}$. Furthermore, for any element $x \in F$, we have $B_{F} \cap Y \subseteq B_{x} \cap Y$, and so $B_{x} \cap Y \in \mathscr{U}$. By definition, it follows that $F \subseteq A_{Y, \mathscr{U}}$, that is, $A_{Y, \mathscr{U}} \in B_{F} \subseteq \cup \mathcal{B}^{\prime}=U$. Now, suppose that 
$H=X \backslash U$ (thus $Y \backslash U \in \mathscr{U}$ ). We want to show that $A_{Y, \mathscr{U}} \in X \backslash U$. Assume, by contradiction, that $A_{Y, \mathscr{U}} \in B_{F}$, for some $B_{F} \in \mathcal{B}^{\prime}$. It follows immediately that $B_{F} \cap Y \in \mathscr{U}$ and, finally, $\emptyset=\left(B_{F} \cap Y\right) \cap(Y \backslash U) \in \mathscr{U}$; a contradiction.

3.9. Proposition. We preserve the notation of Proposition 3.1 and, now, let $\operatorname{Zar}(K(T))$ and $\operatorname{Zar}(K)$ be endowed with the constructible topology. Then, the canonical (surjective) map $\varphi: \operatorname{Zar}(K(T))^{\text {cons }} \rightarrow \operatorname{Zar}(K)^{\text {cons }}$ is continuous and (hence) closed. In particular, $\left.\varphi\right|_{\operatorname{Zar}_{0}(K(T))}$ is a homeomorphism of $\operatorname{Zar}_{0}(K(T))^{\text {cons }}$ onto $\operatorname{Zar}(K)^{\text {cons }}$.

Proof. Let $C$ be a closed subset of $\operatorname{Zar}(K)^{\text {cons }}$, and let $\mathscr{U}$ be an ultrafilter on $\varphi^{-1}(C)$. Set $\varphi^{\prime}:=\left.\varphi\right|_{\varphi^{-1}(C)}: \varphi^{-1}(C) \longrightarrow C$. Then, by [7, Lemma 2.1(4)], the set $\mathscr{V}:=\mathscr{U}_{\varphi^{\prime}}$ is an ultrafilter on $C$. Let $V:=A_{\mathscr{V}} \in \operatorname{Zar}(K)$ and $W:=A_{\mathscr{U}} \in \operatorname{Zar}(K(T))$; then, by a routine argument, it is easy to check that $\varphi(W)=V \in C$. By Proposition 2.3 (2), it follows that $\varphi$ is continuous. Moreover (by [5, Chapter XI, Theorem 2.1]), $\varphi$ is closed, since $\operatorname{Zar}(K(T))^{\text {cons }}$ and $\operatorname{Zar}(K)^{\text {cons }}$ are compact and Hausdorff [7. Theorem 3.4(5)(6)]. Finally, the last statement is a consequence of the fact that the restriction of $\varphi$ to $\operatorname{Zar}_{0}(K(T))$ is bijective.

3.10. Remark. Let $K$ be a field, $T$ an indeterminate over $K$, and set $R_{0}:=$ $\bigcap\{V(T) \mid V \in \operatorname{Zar}(K)\}$. Then, by Propositions $[3.2(2)$ and 3.3 , it follows that

$$
\operatorname{Zar}_{0}(K(T))=\operatorname{Zar}\left(K(T) \mid R_{0}\right) .
$$

In particular, $\operatorname{Zar}_{0}(K(T))$ is a closed subspace of $\operatorname{Zar}(K(T))^{\text {cons }}$, in view of 7 , Theorem 3.4(2)(6)].

A "constructible" version of Corollary [3.6 (2)(3) can also be easily deduced from the previous considerations.

3.11. Corollary. Let $K$ be a field, $A$ any subring of $K, T$ an indeterminate over $K$, and let $\operatorname{Kr}(K \mid A)$ be as in Corollary 3.6.

(1) The canonical map $\varphi: \operatorname{Zar}(K(T) \mid \operatorname{Kr}(K \mid A))^{\text {cons }} \rightarrow \operatorname{Zar}(K \mid A)^{\text {cons }}$, defined by $W \mapsto W \cap K$, is a homeomorphism.

(2) The canonical map $\psi: \operatorname{Spec}(\operatorname{Kr}(K \mid A))^{\text {cons }} \rightarrow \operatorname{Zar}(K \mid A)^{\text {cons }}$, defined by $Q \mapsto$ $\operatorname{Kr}(K \mid A)_{Q} \cap K$, is a homeomorphism. In particular, $\operatorname{Zar}(K \mid A)^{\text {cons }}$ is a spectral space canonically homeomorphic to the prime spectrum of the absolutely flat ring canonically associated to the $K$-function ring $\operatorname{Kr}(K \mid A)$.

Proof. (1) As observed in the proof of Corollary [3.6)(1), $\operatorname{Kr}(K \mid A)$ is a $K$-function ring; hence, this statement follows from Proposition 3.9.

(2) is a consequence of (1), [7, Theorem 3.8 and Remark 3.9] and [13, Propositions 5 and 6 , and Theorem 8].

\section{Some APPLiCATIONS}

Let $K$ be a field, and let $A$ be a subring of $K$. In this section, we constantly use that on the space $\operatorname{Zar}(K \mid A)$, the contructible topology coincides with the ultrafilter topology (Remark 2.5) and we give some applications of the results of the previous sections to the representations of integrally closed domains as intersections of valuation overrings. 
4.1. Proposition. Let $K$ be a field, $A$ be a subring of $K$ and $U$ be a subset of $Z:=\operatorname{Zar}(K \mid A)$. Let $Y^{\prime}$ and $Y^{\prime \prime}$ be two subsets of a given subset $U$ of $Z$ and assume that their closures in $U$, with the subspace topology induced by the constructible topology of $Z$, coincide, i.e., $C l^{\text {cons }}\left(Y^{\prime}\right) \cap U=C l^{\text {cons }}\left(Y^{\prime \prime}\right) \cap U$. Then,

$$
\bigcap\left\{V^{\prime} \mid V^{\prime} \in Y^{\prime}\right\}=\bigcap\left\{V^{\prime \prime} \mid V^{\prime \prime} \in Y^{\prime \prime}\right\} .
$$

In particular, for each subset $Y$ of $Z$,

$$
\bigcap\{V \mid V \in Y\}=\bigcap\left\{W \mid W \in \operatorname{Cl}^{\text {cons }}(Y)\right\} .
$$

Proof. Assume, by contradiction, that there is an element

$$
x_{0} \in \bigcap\left\{V^{\prime} \mid V^{\prime} \in Y^{\prime}\right\} \backslash \bigcap\left\{V^{\prime \prime} \mid V^{\prime \prime} \in Y^{\prime \prime}\right\},
$$

and pick a valuation domain $V_{0} \in Y^{\prime \prime}$ such that $x_{0} \notin V_{0}$. By [7, Theorem 3.4(2)], the set $\Omega:=U \backslash B_{x_{0}}$ is an open subset of $U$, with respect to the subspace topology induced by $Z^{\text {cons }}$, and it contains $V_{0}$. Since $V_{0} \in Y^{\prime \prime} \subseteq C l^{\text {cons }}\left(Y^{\prime \prime}\right) \cap U=C l^{\text {cons }}\left(Y^{\prime}\right) \cap$ $U$ and $V_{0} \notin Y^{\prime}$, then $\Omega \cap Y^{\prime}$ is nonempty. This implies that there exists a valuation domain $V^{\prime} \in Y^{\prime}$ such that $x_{0} \notin V^{\prime}$; a contradiction.

4.2. Remark. Note that the previous proposition is stated very generally using a "relative-type" formulation. However, it is clear that, if we take any two subsets $Y^{\prime}$ and $Y^{\prime \prime}$ of $Z$, the rôle of $U$ can be played by any subset of $Z$ (including $Z$ ) containing $Y^{\prime} \cup Y^{\prime \prime}$.

Let $\Sigma$ be a collection of subrings of a field $K$, having $K$ as the quotient field. We say that $\Sigma$ is locally finite if each nonzero element of $K$ is noninvertible in at most finitely many of the rings belonging to $\Sigma$.

The following easy result will provide a class of integral domains for which the equality $\bigcap\left\{V^{\prime} \mid V^{\prime} \in Y^{\prime}\right\}=\bigcap\left\{V^{\prime \prime} \mid V^{\prime \prime} \in Y^{\prime \prime}\right\}$ does not imply, in general, that $C l^{\text {cons }}\left(Y^{\prime}\right)=C l^{\text {cons }}\left(Y^{\prime \prime}\right)$.

4.3. Lemma. Let $K$ be a field and $A$ be a subring of $K$. If $\Sigma$ is an infinite and locally finite subset of $Z:=\operatorname{Zar}(K \mid A)$, then $\mathrm{Cl}^{\text {cons }}(\Sigma)=\Sigma \cup\{K\}$.

Proof. By Proposition 2.3(2) and [7, Remark 3.2], it is enough to show that $K=$ $A_{\mathscr{U}}\left(=\left\{x \in K \mid B_{x} \cap \Sigma \in \mathscr{U}\right\}\right)$, for every nontrivial ultrafilter $\mathscr{U}$ on $\Sigma$. By contradiction, assume that there exists an element $x_{0} \in K \backslash A_{\mathscr{U}}$. Then $\Sigma \backslash B_{x_{0}} \in \mathscr{U}$, and so it is infinite, since $\mathscr{U}$ is nontrivial (an ultrafilter containing a finite set is trivial). This implies that $x_{0}$ is noninvertibile in infinitely many valuation domains belonging to $\Sigma$; a contradiction.

As a consequence of the previous lemma, we have that if an integral domain admits two distinct infinite and locally finite representations as intersection of valutation domains, then the converse of Proposition 4.1 does not hold. An explicit example is given next.

4.4. Example. Let $k$ be a field and let $T_{1}, T_{2}, T_{3}$ be three indeterminates. Let $B$ be the two-dimensional, local domain $k\left(T_{3}\right)\left[T_{1}, T_{2}\right]_{\left(T_{1}, T_{2}\right)}$ with maximal ideal $M_{B}:=\left(T_{1}, T_{2}\right) k\left(T_{3}\right)\left[T_{1}, T_{2}\right]_{\left(T_{1}, T_{2}\right)}$, i.e., $B=k\left(T_{3}\right)+M_{B}$. Now, let $V$ be (the rank 1 discrete) valuation domain defined by $V:=k\left[T_{3}\right]_{\left(T_{3}\right)}$ and let $A$ be the pullback domain given by

$$
A:=V+M_{B}=k\left[T_{3}\right]_{\left(T_{3}\right)}+\left(T_{1}, T_{2}\right) k\left(T_{3}\right)\left[T_{1}, T_{2}\right]_{\left(T_{1}, T_{2}\right)} .
$$


Our goal is to represent $A$ as a locally finite intersection of valuation domains in two different ways. In fact, we can use one description to generate an infinite number of different such representations.

Note that $B$ can be represented as an intersection of DVR's which are obtained by localizing at its height-one primes, i.e., $B=\bigcap\left\{B_{P} \mid P \in \operatorname{Spec}(B)\right.$, ht $\left.(P)=1\right\}$. It is well known that this collection is locally finite. Now, note that $A$ is a local domain with maximal ideal $N_{A}:=T_{3} k\left[T_{3}\right]_{\left(T_{3}\right)}+\left(T_{1}, T_{2}\right) k\left(T_{3}\right)\left[T_{1}, T_{2}\right]_{\left(T_{1}, T_{2}\right)}$. Choose any valuation overring $W$ of $A$ such that $M_{W}$ (the maximal ideal of $W$ ) is generated by $T_{3}$ and lies over the maximal ideal of $A$. It is easy to see that many such valuation domains of the field $k\left(T_{1}, T_{2}, T_{3}\right)$ exist (e.g., let $W^{\prime}$ be a valuation overring of $B$ with maximal ideal $M^{\prime}$ lying over $M_{B}$ and such that the residue field $W^{\prime} / M^{\prime}$ is canonically isomorphic to $k\left(T_{3}\right)$, which is the residue field $B / M_{B}$, then the domain $V+M^{\prime}$, with $V$ as in the previous paragraph, can serve as the desired domain $W)$. Now, the intersection $R:=\bigcap\left\{B_{P} \mid P \in \operatorname{Spec}(B), \operatorname{ht}(P)=1\right\} \bigcap W$ is clearly a locally finite intersection. We claim that any choice, as above, of the domain $W$ will yield $R=A$.

To prove our claim, we note first that it is obvious that $R$ is an overring of $A$. So, we need to prove that $R \subseteq A$. Observe that the ideal $M_{B}$ is an ideal of $A$ as well as of $B$. It follows easily that $M_{B}$ is a prime ideal of $R$, since $R \subset B$. Then, given an element $r \in R$, we can write $r=\psi+f$, where $\psi \in k\left(T_{3}\right)$ and $f \in M_{B}$. However, $f \in M_{B} \subseteq W$ and so $\psi \in W$. It is clear that $W \cap k\left(T_{3}\right)=V$. It follows that $\psi \in V$ and so $r \in A$. Hence, we have proven that $R \subseteq A$.

The following proposition is the key step in proving the main results of the section.

4.5. Proposition. Let $A$ be a Prüfer domain and $K$ be the quotient field of $A$. Let $Y$ be a subset of $Z:=\operatorname{Zar}(K \mid A)$ such that $A=\bigcap\{V \mid V \in Y\}$, and let $\gamma: \operatorname{Zar}(K \mid A) \longrightarrow \operatorname{Spec}(A)$ be the canonical map (defined by sending a valuation domain $V \in \operatorname{Zar}(K \mid A)$ into its center in $A)$. Then, $\gamma^{-1}(\operatorname{Max}(A)) \subseteq C l^{\text {cons }}(Y)$.

Proof. Let $M$ be a maximal ideal of $A$. Since $A$ is a Prüfer domain, the $t$-operation on $A$ coincides with the identity [15, Theorem 22.1(3)], thus obviously $M$ is a $t$ maximal $t$-ideal of $A$. We are now able to apply [1, Proposition 2.8(ii)] and, so, there exists an ultrafilter $\mathscr{U} \in \beta(Y)$ such that

$$
M=\left\{x \in A \mid \gamma^{-1}(V(x)) \cap Y \in \mathscr{U}\right\} .
$$

On the other hand, the collection of sets

$$
\mathscr{V}:=\left\{X^{\prime} \subseteq \gamma(Y) \mid \gamma^{-1}\left(X^{\prime}\right) \cap Y \in \mathscr{U}\right\}
$$

is an ultrafilter on $\gamma(Y)$ (precisely, with the notation of [7, Lemma 2.1(4)], $\mathscr{V}=\mathscr{U}_{\gamma}$, where for simplicity we have still denoted the map $\left.\gamma\right|_{Y}: Y \rightarrow \gamma(Y)$ by $\left.\gamma\right)$ and, moreover,

$$
\begin{aligned}
P_{\mathscr{V}} & :=\{x \in A \mid V(x) \cap \gamma(Y) \in \mathscr{V}\} \\
& =\left\{x \in A \mid \gamma^{-1}(V(x) \cap \gamma(Y)) \cap Y \in \mathscr{U}\right\} \\
& =\left\{x \in A \mid \gamma^{-1}(V(x)) \cap Y \in \mathscr{U}\right\}=M .
\end{aligned}
$$

Moreover, if $A_{\mathscr{U}}$ is the ultrafilter limit valuation domain of $K$ associated to $\mathscr{U} \in$ $\beta(Y)$ (i.e., $A_{\mathscr{U}}=\left\{x \in K \mid B_{x} \cap Y \in \mathscr{U}\right\}$, Corollary [3.8), then by [1, Proposition 2.10(i)], we have $\gamma\left(A_{\mathscr{U}}\right)=P_{\mathscr{V}}=M$. Therefore, $\operatorname{Max}(A) \subseteq C l^{\text {cons }}(\gamma(Y))$. Since $\gamma$ is continuous and closed with respect to the constructible topology [7, Theorem 3.8], it follows that $C l^{\text {cons }}(\gamma(Y))=\gamma\left(C l^{\text {cons }}(Y)\right)$. 
Moreover, since $A$ is a Prüfer domain, by [4, Proposition 2.2], $\gamma$ is also injective and, hence, $\gamma^{-1}(\operatorname{Max}(A)) \subseteq C l^{\text {cons }}(Y)$.

Let $A$ be a domain, $K$ be the quotient field of $A$, and $T$ be an indeterminate over $K$. For each subset $Y$ of $Z:=\operatorname{Zar}(K \mid A)$, we set

$$
Y_{0}:=\{V(T) \mid V \in Y\}, \quad Y^{\uparrow}:=\{V \in Z \mid V \supseteq W, \text { for some } W \in Y\},
$$

where $Y^{\uparrow}$ coincides with $Y^{\text {gen }}$ the Zariski-generic closure of $Y$, i.e., the generic closure of $Y$ in $Z$, with respect to the Zariski topology (since $V \preccurlyeq W$ (in $Z^{\text {zar }}$ ): $\Leftrightarrow$ $V \supseteq W)$. Recall that, in Corollary [3.6(1), we introduced a general form of the Kronecker function ring, by setting $\operatorname{Kr}(K \mid A)=\bigcap\{V(T) \mid V \in Z\}=\bigcap Z_{0}=$ : $\operatorname{Kr}(Z)$. Now, we can extend this notion for $Y \subseteq Z$, by setting

$$
\operatorname{Kr}(Y):=\bigcap Y_{0}=\bigcap\{V(T) \mid V \in Y\}
$$

which is called the $K$-function ring associated to $Y$. We recall that an integrally closed domain $A$ is a vacant domain if, for each $Y \subseteq Z$ such that $A=\bigcap Y$, then $\operatorname{Kr}(Y)=\operatorname{Kr}(Z)$ [6, Definition 2.1.11].

4.6. Theorem. Let $K$ be a field and $C$ a closed subset of $\operatorname{Zar}(K)^{\text {cons }}$. Let $\left(C^{\uparrow}\right)_{0}=$ $\left\{W(T) \mid W \in C^{\uparrow}\right\}$. Then, $\operatorname{Zar}(K(T) \mid \operatorname{Kr}(C))=\left(C^{\uparrow}\right)_{0}$.

Proof. The inclusion $\supseteq$ is obvious. For the converse, let $\widetilde{W} \in \operatorname{Zar}(K(T) \mid \operatorname{Kr}(C))$. By Proposition 3.3 , we can suppose that $\widetilde{W}=W(T)$, for some $W \in \operatorname{Zar}(K)$. We want to show that $W \supseteq V$, for some $V \in C$. Let $\varphi: \operatorname{Zar}(K(T)) \rightarrow \operatorname{Zar}(K)$ be the canonical map (Proposition 3.1). Since $\varphi{\mid \operatorname{Zar}_{0}(K(T))}: \operatorname{Zar}_{0}(K(T))^{\text {cons }} \rightarrow \operatorname{Zar}(K)^{\text {cons }}$ is a homeomorphism (Proposition 3.9), then the set

$$
\left(\varphi \mid \operatorname{Zar}_{0}(K(T))\right)^{-1}(C)=\{V(T) \mid V \in C\}=C_{0}
$$

is closed both in $\operatorname{Zar}_{0}(K(T))^{\text {cons }}$ and $\operatorname{Zar}(K(T))^{\text {cons }}$ (Remark 3.10). Consider the natural map $\gamma: \operatorname{Zar}(K(T) \mid \operatorname{Kr}(C))^{\text {cons }} \rightarrow \operatorname{Spec}(\operatorname{Kr}(C))^{\text {cons }}$, defined by sending a valuation overring of $\operatorname{Kr}(C)$ into its center on $\operatorname{Kr}(C)$. Since the Kronecker function ring $\operatorname{Kr}(C)$ is, in particular, a Prüfer domain with quotient field $K(T)$ (Proposition 3.2 (3)) then, from Proposition 4.5, it follows immediately that $\gamma^{-1}(\operatorname{Max}(\operatorname{Kr}(C))) \subseteq$ $C_{0}$. Set $A(C):=\bigcap\{V \mid V \in C\}$. Now, by Zorn's Lemma, we can find a minimal valuation overring of $\operatorname{Kr}(C)$ which, by Proposition 3.3, is of the form $V^{\prime}(T)$, for some $V^{\prime} \in \operatorname{Zar}(K \mid A(C))$, such that $W(T) \supseteq V^{\prime}(T)$. Then, by applying 15, Corollary 19.7] (and, again, Proposition [3.3), we have $\operatorname{Zar}_{\min }(\operatorname{Kr}(C)) \subseteq \gamma^{-1}(\operatorname{Max}(\operatorname{Kr}(C))$ ). Since, by what we observed above, $\gamma^{-1}(\operatorname{Max}(\operatorname{Kr}(C))) \subseteq C_{0}$, then $V^{\prime}(T) \in C_{0}$.

4.7. Remark. Note that, with the notation and assumptions of Theorem 4.6,

$$
\left(C^{\uparrow}\right)_{0}=\left(C_{0}\right)^{\uparrow}:=\{\widetilde{W} \in \operatorname{Zar}(K(T)) \mid \widetilde{W} \supseteq V(T) \text {, for some } V \in C\} .
$$

As a matter of fact, $\{\widetilde{W} \in \operatorname{Zar}(K(T)) \mid \widetilde{W} \supseteq V(T)$, for some $V \in C\}=\{\widetilde{W} \in$ $\operatorname{Zar}(K(T) \mid \operatorname{Kr}(C)) \mid \widetilde{W} \supseteq V(T)$, for some $V \in C\}$. By Proposition 3.3. we have $\operatorname{Zar}(K(T) \mid \operatorname{Kr}(C))=\operatorname{Zar}_{0}(K(T) \mid \operatorname{Kr}(C))$, thus $\left(C_{0}\right)^{\uparrow}=\{W(T) \in \operatorname{Zar}(K(T)) \mid W \in$ $\operatorname{Zar}(K), W(T) \supseteq V(T)$, for some $V \in C\}=\left(C^{\uparrow}\right)_{0}$.

We recall some properties of semistar operations. Let $A$ be an integral domain and let $K$ be the quotient field of $A$. We denote by $\overline{\boldsymbol{F}}(A)$ the set of all the nonzero $A$-submodules of $K$, and by $\boldsymbol{f}(A)$ the set of all the nonzero finitely generated $A$ submodules of $K$. A map $\star: \overline{\boldsymbol{F}}(A) \rightarrow \overline{\boldsymbol{F}}(A), E \mapsto E^{\star}$, is called a semistar operation 
on $A$ if, for each $0 \neq x \in K$ and for all $E, F \in \overline{\boldsymbol{F}}(A)$, the following properties hold: $\left(\star_{1}\right)(x E)^{\star}=x E^{\star} ; \quad\left(\star_{2}\right) E \subseteq F \Rightarrow E^{\star} \subseteq F^{\star} ; \quad\left(\star_{3}\right) E \subseteq E^{\star}$ and $\left(E^{\star}\right)^{\star}=E^{\star}$.

A semistar operation of finite type $\star$ on $A$ is a semistar operation such that, for every $E \in \overline{\boldsymbol{F}}(A)$,

$$
E^{\star}=E^{\star_{f}}:=\bigcup\left\{F^{\star} \mid F \in \boldsymbol{f}(A), F \subseteq E\right\} .
$$

An e.a.b. semistar operation $\star$ on $A$ is a semistar operation such that, for all $F, G, H \in \boldsymbol{f}(A),(F G)^{\star} \subseteq(F H)^{\star}$ implies $G^{\star} \subseteq H^{\star}$.

A valuation domain $V \in \operatorname{Zar}(K \mid A)$ is called a $\star$-valuation overring of $A$ if $F^{\star} \subseteq$ $F V$, for each $F \in \boldsymbol{f}(A)$. We denote by $\operatorname{Zar}^{\star}(K \mid A)$ the collection of all the $\star-$ valutation overring of $A$.

Important classes of examples of semistar operations are obtained as follows. Let $\mathcal{S}$ be a nonempty family of overrings of $A$. Then the map $\bigwedge_{\mathcal{S}}: \overline{\boldsymbol{F}}(A) \rightarrow \overline{\boldsymbol{F}}(A)$, $E \mapsto \bigcap\{E S \mid S \in \mathcal{S}\}$, defines a semistar operation on $A$ [9, Theorem 1.2(C)]. In particular, given a nonempty subset $Y$ of $\operatorname{Zar}(K \mid A)$, the semistar operation $\bigwedge_{Y}$ is e.a.b., by [12, Proposition 7]. We say that a semistar operation $\star$ on $A$ is complete if $\star=b(\star):=\bigwedge_{\operatorname{Zar}^{\star}(K \mid A)}$. For any semistar operation $\star$ on $A$, it is easily seen that $F^{b(\star)} V=F^{\star} V$, for each $F \in \boldsymbol{f}(A)$ and $V \in \operatorname{Zar}^{\star}(K \mid A)$. Thus, $b(b(\star))=b(\star)$ and $b(\star)$ is a complete semistar operation. The $b$-operation on $A$ is the e.a.b. semistar operation defined by $b:=\bigwedge_{\operatorname{Zar}(K \mid A)}$ and, obviously, $b \leq b(\star)$ (i.e., $E^{b} \subseteq E^{b(\star)}$ for each $E \in \overline{\boldsymbol{F}}(A))$ for all semistar operations $\star$ on $A$.

4.8. Remark. Let $K$ be a field and let $A$ be a subring of $K$. If $Y$ is a nonempty subset of $\operatorname{Zar}(K \mid A)$, then it is immediately seen that $\bigwedge_{Y}=\bigwedge_{Y \uparrow}$. Moreover, if $Y$ is a quasi-compact subset of $\operatorname{Zar}(K \mid A)^{\mathrm{zar}}$, the subset $Y_{\min }$, consisting of the minimal elements of $Y$, is nonempty and it is easy to see that $\bigwedge_{Y \uparrow}=\bigwedge_{Y}=\bigwedge_{Y_{\min }}$.

Let $T$ be an indeterminate over $A$ and $f \in A[T]$. We shall denote by $\boldsymbol{c}(f)$ the content of the polynomial $f$. If $\star$ is an e.a.b. semistar operation on $A$,

$$
\operatorname{Kr}(A, \star):=\left\{f / g \in K(T) \mid f, g \in A[T], g \neq 0, \text { and } \boldsymbol{c}(f)^{\star} \subseteq \boldsymbol{c}(g)^{\star}\right\}
$$

is called the $\star$-Kronecker function ring of $A$. It is well known that $\operatorname{Kr}(A, \star)$ is a Bézout domain with quotient field $K(T)$ [10, Theorems 5.1 and 3.11(3)]. Note that, if $Y$ is a subset of $\operatorname{Zar}(K \mid A)$, by [10, Corollary 3.8], the $\bigwedge_{Y}$-Kronecker function ring of $A, \operatorname{Kr}\left(A, \bigwedge_{Y}\right)$, coincides with the $K$-function $\operatorname{ring} \operatorname{Kr}(Y)$ (introduced just before Theorem 4.6).

Now, we give an application of the ultrafilter topology for characterizing when two e.a.b. semistar operations of finite type are equal.

4.9. Theorem. Let $A$ be an integral domain with quotient field $K$ and $Y^{\prime}, Y^{\prime \prime} \subseteq$ $\operatorname{Zar}(K \mid A)$. Then, the following conditions are equivalent:

(i) $\left(\bigwedge_{Y^{\prime}}\right)_{f}=\left(\bigwedge_{Y^{\prime \prime}}\right)_{f}$.

(ii) The sets $C l^{\text {cons }}\left(Y^{\prime}\right), C l^{\text {cons }}\left(Y^{\prime \prime}\right)$ have the same Zariski-generic closure in $\operatorname{Zar}(K \mid A)$, i.e., $C l^{\text {cons }}\left(Y^{\prime}\right)^{\uparrow}=C l^{\text {cons }}\left(Y^{\prime \prime}\right)^{\uparrow}$.

Proof. Let $T$ be an indeterminate over $K$. By [10, Remark 3.5(b)], it is enough to show that condition (ii) is equivalent to the following:

(i') $\operatorname{Kr}\left(A, \bigwedge_{Y^{\prime}}\right)=\operatorname{Kr}\left(A, \bigwedge_{Y^{\prime \prime}}\right)$.

(ii) $\Rightarrow\left(\mathrm{i}^{\prime}\right)$. Assume that the equality $C l^{\text {cons }}\left(Y^{\prime}\right)^{\uparrow}=C l^{\text {cons }}\left(Y^{\prime \prime}\right)^{\uparrow}$ holds. Keeping in mind the notation introduced before Theorem 4.6 and applying Corollary 3.11(1), 
it easily follows that, inside $\operatorname{Zar}(K(T)), C l^{\text {cons }}\left(Y_{0}^{\prime}\right)^{\uparrow}=C l^{\text {cons }}\left(Y_{0}^{\prime \prime}\right)^{\uparrow}$. By using Proposition 4.1 and Remark 4.8 , we have

$$
\begin{aligned}
\bigcap Y_{0}^{\prime} & =\bigcap C l^{\text {cons }}\left(Y_{0}^{\prime}\right)=\bigcap C l^{\text {cons }}\left(Y_{0}^{\prime}\right)^{\uparrow}=\bigcap C l^{\text {cons }}\left(Y_{0}^{\prime \prime}\right)^{\uparrow} \\
& =\bigcap C l^{\text {cons }}\left(Y_{0}^{\prime \prime}\right)=\bigcap Y_{0}^{\prime \prime},
\end{aligned}
$$

and thus $\operatorname{Kr}\left(A, \bigwedge_{Y^{\prime}}\right)=\operatorname{Kr}\left(A, \bigwedge_{Y^{\prime \prime}}\right)$, in view of [10, Corollary 3.8].

$\left(\mathrm{i}^{\prime}\right) \Rightarrow(\mathrm{ii})$. Set $B:=\operatorname{Kr}\left(A, \bigwedge_{Y^{\prime}}\right)=\operatorname{Kr}\left(A, \bigwedge_{Y^{\prime \prime}}\right)$. By using [10, Corollary 3.8], Proposition 4.1. Theorem 4.6 and Remark 4.7, it follows that

$$
C l^{\text {cons }}\left(Y_{0}^{\prime}\right)^{\uparrow}=\operatorname{Zar}(K(T) \mid B)=C l^{\text {cons }}\left(Y_{0}^{\prime \prime}\right)^{\uparrow},
$$

and thus the conclusion is clear, again by Corollary $3.11(1)$.

4.10. Corollary. Let $A$ be an integrally closed domain. Then, the following conditions are equivalent:

(i) A is a vacant domain.

(ii) For each representation $Y \subseteq \operatorname{Zar}(K \mid A)$ of $A$ (i.e., $\bigcap Y=A$ ), we have $\mathrm{Cl}^{\text {cons }}(Y)^{\uparrow}=\operatorname{Zar}(K \mid A)$.

Proof. Set $Z:=\operatorname{Zar}(K \mid A)$.

(i) $\Rightarrow$ (ii). Assume $A$ is vacant and take a subset $Y \subseteq Z$ such that $\bigcap Y=A$. By 10, Proposition 3.3], we have $\operatorname{Kr}\left(A, \bigwedge_{Y}\right)=\operatorname{Kr}(Y)=\operatorname{Kr}(Z)=\operatorname{Kr}(A, b)$, and thus

$$
\left(\bigwedge_{Y}\right)_{f}=b=\bigwedge_{Z}=\left(\bigwedge_{Z}\right)_{f} .
$$

The conclusion immediately follows from Theorem 4.9

(ii) $\Rightarrow$ (i). Take a subset $Y$ of $\operatorname{Zar}(K \mid A)$ such that $\bigcap Y=A$. By assumption and Theorem 4.9, it follows that $\left(\bigwedge_{Y}\right)_{f}=\left(\bigwedge_{Z}\right)_{f}=\bigwedge_{Z}=b$, and thus $\operatorname{Kr}(Y)=$ $\operatorname{Kr}\left(A, \bigwedge_{Y}\right)=\operatorname{Kr}\left(A, \bigwedge_{Z}\right)=\operatorname{Kr}(Z)$. This proves that $A$ is vacant.

From the previous theorem, we immediately deduce the following corollary.

4.11. Corollary. Let $A$ be an integrally closed domain. If each representation of $A$ is dense in $\operatorname{Zar}(K \mid A)^{\text {cons }}$, then $A$ is a vacant domain.

4.12. Example. Let $K$ be a field and let $T_{1}, T_{2}$ be two indeterminates over $K$. Consider the pseudo-valuation domain $A:=K+T_{2} K\left(T_{1}\right)\left[T_{2}\right]_{\left(T_{2}\right)}$ with associated valuation domain $V:=K\left(T_{1}\right)\left[T_{2}\right]_{\left(T_{2}\right)}$ of $K\left(T_{1}, T_{2}\right)$. Let $p: V \rightarrow K\left(T_{1}\right)$ be the canonical projection of $V$ onto its residue field $K\left(T_{1}\right)$ and so $A=p^{-1}(K)$. Then, by [15. Exercise 12, page 409], the domain $A$ is a vacant domain. It is easily seen that the set $C:=\left\{p^{-1}\left(W^{\prime}\right) \mid W^{\prime} \in \operatorname{Zar}\left(K\left(T_{1}\right) \mid K\right)\right\} \subset \operatorname{Zar}\left(K\left(T_{1}, T_{2}\right) \mid A\right)$ is a representation of $A$, and that it is closed, with respect to the constructible topology of $\operatorname{Zar}\left(K\left(T_{1}, T_{2}\right) \mid A\right)$, since $C=\left\{W \in \operatorname{Zar}\left(K\left(T_{1}, T_{2}\right) \mid A\right) \mid W \subseteq V\right\}=$ $\bigcap_{z \in K\left(T_{1}, T_{2}\right) \backslash V}\left(\operatorname{Zar}\left(K\left(T_{1}, T_{2}\right) \mid A\right) \backslash B_{z}\right)=C l^{\mathrm{zar}}(\{V\})$. Thus, the converse of the previous Corollary 4.11 does not hold in general.

Note that this example also shows that, in the statement of Theorem 4.6, we need to consider $C^{\uparrow}$ and not just $C$, since in this case $\operatorname{Zar}\left(K\left(T ; T_{1}, T_{2}\right) \mid \operatorname{Kr}\left(A, \bigwedge_{C}\right)\right)=$ $\operatorname{Zar}\left(K\left(T ; T_{1}, T_{2}\right) \mid \operatorname{Kr}(C)\right)=\left(C^{\uparrow}\right)_{0} \supsetneq C_{0}$.

Now, we prove that the property of being "complete" for a semistar operation can be characterized by a "compactness" property for a suitable subspace of the Zariski-Riemann surface. 
4.13. Theorem. Let $A$ be an integral domain with quotient field $K$ and $\star$ be $a$ semistar operation on $A$. Then, the following conditions are equivalent:

(i) $\star$ is e.a.b. of finite type.

(ii) $\star$ is complete.

(iii) There exists a closed subset $Y$ of $\operatorname{Zar}(K \mid A)^{\text {cons }}$ such that $Y=Y^{\uparrow}$ and $\star=\bigwedge_{Y}$.

(iv) There exists a compact subspace $Y^{\prime}$ in $\operatorname{Zar}(K \mid A)^{\text {cons }}$ such that $\star=\bigwedge_{Y^{\prime}}$.

(v) There exists a quasi-compact subspace of $Y^{\prime \prime}$ of $\operatorname{Zar}(K \mid A)^{\text {zar }}$ such that $\star=$ $\bigwedge_{Y^{\prime \prime}}$.

Proof. (i) $\Leftrightarrow$ (ii) depends on the fact that if $\star$ is e.a.b., then $\star_{f}=b(\star)$ [12, Proposition 9].

Let $T$ be an indeterminate on $K$ and let $\varphi: \operatorname{Zar}(K(T)) \longrightarrow \operatorname{Zar}(K)$ be the canonical surjective map, defined in Proposition 3.1 .

(ii) $\Rightarrow$ (iv). By [11, Theorem 3.5], $\operatorname{Zar}^{\star}(K \mid A)=\varphi(\operatorname{Zar}(K(T) \mid \operatorname{Kr}(A, \star)))$, and thus (by Proposition [3.9) it is closed in $\operatorname{Zar}(K \mid A)^{\text {cons }}$ or, equivalently, compact in the compact Hausdorff space $\operatorname{Zar}(K \mid A)^{\text {cons }}$. Then, the conclusion follows by taking $Y^{\prime}:=\operatorname{Zar}^{\star}(K \mid A)$ (since, by definition, $\left.b(\star)=\bigwedge_{\operatorname{Zar}^{\star}(K \mid A)}\right)$.

(iv) $\Rightarrow$ (ii). As observed above, the compact subspaces of $\operatorname{Zar}(K \mid A)^{\text {cons }}$ are exactly the closed subsets. Take a closed set $Y^{\prime}$ of $\operatorname{Zar}(K \mid A)^{\text {cons }}$ such that $\star=\bigwedge_{Y^{\prime}}$. Set $Y^{\prime \uparrow}:=\left\{W \in \operatorname{Zar}(K \mid A) \mid W \supseteq V\right.$, for some $\left.V \in Y^{\prime}\right\}$. By [10, Corollary 3.8], we have $\operatorname{Kr}\left(A, \bigwedge_{Y^{\prime}}\right)=\bigcap\left\{V(T) \mid V \in Y^{\prime}\right\}=: \operatorname{Kr}\left(Y^{\prime}\right)$. On the other hand, since $Y^{\prime}$ is closed, by Theorem 4.6. it follows that $\operatorname{Zar}\left(K(T) \mid \operatorname{Kr}\left(A, \bigwedge_{Y^{\prime}}\right)\right)=\left(Y^{\prime \uparrow}\right)_{0}=$ $\left\{W(T) \mid W \in Y^{\prime \uparrow}\right\}$. Therefore, as above (by [11, Theorem 3.5]), $\operatorname{Zar}^{\wedge} \wedge_{Y}(K \mid A)=$ $\varphi\left(\operatorname{Zar}\left(K(T) \mid \operatorname{Kr}\left(A, \bigwedge_{Y}\right)\right)\right)=Y^{\uparrow}$ and so $Y^{\uparrow}$ is also a closed subspace of $\operatorname{Zar}(K \mid A)^{\text {cons }}$. Since by definition $b\left(\bigwedge_{Y}\right)=\bigwedge_{\mathrm{Zar} \wedge_{Y}(K \mid A)}=\bigwedge_{Y^{\uparrow}}$, then the conclusion is immediate, by Remark 4.8 .

(iii) $\Rightarrow$ (iv) is trivial since, as observed above, closed coincides with compact in $\operatorname{Zar}(K \mid A)^{\text {cons }}$.

(iv) $\Rightarrow(\mathrm{v})$ is obvious, by [7, Theorem 3.4(1)].

$(\mathrm{v}) \Rightarrow$ (iii). Take a set $Y^{\prime \prime}$ as stated in (v). Then, (iii) follows immediately from Proposition 2.6 and Remark 4.8, by taking $Y:=Y^{\prime \prime \uparrow}$.

4.14. Corollary. Let $A$ be an integral domain and $K$ its quotient field. Let $Y$ be a subset of $\operatorname{Zar}(K \mid A)$ and set $\widehat{Y}:=C l^{\text {cons }}(Y)^{\uparrow}$. Then, $\left(\bigwedge_{Y}\right)_{f}=\bigwedge_{\widehat{Y}}=\bigwedge_{C l^{c o n s}(Y)}$.

Proof. In view of Proposition 2.6. $\widehat{Y}$ is closed, with respect to the constructible topology. Thus $\bigwedge_{\widehat{Y}}$ is of finite type, by Theorem 4.13, and hence the equality $\left(\bigwedge_{Y}\right)_{f}=\bigwedge_{\widehat{Y}}$ follows immediately by Theorem 4.9. since $\mathrm{Cl}^{\text {cons }}(Y)^{\uparrow}=$ $\widehat{Y}^{\uparrow}\left(=C l^{\text {cons }}\left(\widehat{Y}^{\uparrow}\right)\right)$. Moreover, the semistar operation $\bigwedge_{C l^{\text {cons }}(Y)}$ is of finite type, by Theorem 4.13, and thus the last equality follows by applying Theorem 4.9 .

The next example illustrates the possibility that the sets $Y, Y^{\prime}$ and $Y^{\prime \prime}$ in Theorem 4.13 can form a proper chain of sets.

4.15. Example. Let $k$ be a field and let $T_{1}, T_{2}$ be two indeterminates over $k$. Let $A$ be the two-dimensional, integrally closed, local domain $k\left[T_{1}, T_{2}\right]_{\left(T_{1}, T_{2}\right)}$ with quotient field $K:=k\left(T_{1}, T_{2}\right)$. Let $\star$ be the $b$-operation on $A$. It is well known that the $b$-operation is an e.a.b. operation of finite type. Hence, it satisfies the equivalent conditions of Theorem 4.13. Our goal is to show that there is a great 
deal of flexibility in the choice of the sets $Y, Y^{\prime}$ and $Y^{\prime \prime}$ in the theorem. First, note that if the valuation domains in $\operatorname{Zar}(K \mid A)$ are ordered by inclusion, then any chain is finite [15, Corollary 30.10] and, hence, obviously there are minimal elements. Any such minimal valuation overring $V$ will necessarily have maximal ideal $M_{V}$ lying over the maximal ideal $\left(T_{1}, T_{2}\right)$ of $A$. The standard definition of the $b$-operation involves extending an ideal (or, more generally a sub- $A$-module of $K$ ) to all valuation overrings. It is clearly sufficient to extend to just those valuation overrings that are minimal. So, any subcollection of $\operatorname{Zar}(K \mid A)$ which contains all the minimal elements will generate the $b$-operation. It is not clear that the collection of minimal valuation overrings is closed under the Zariski or the constructible topology.

- Consider the members of $\operatorname{Zar}(K \mid A)$ which do not contain the elements $\frac{1}{T_{1}}, \frac{1}{T_{2}}$. This is a closed, quasi-compact subset of $\operatorname{Zar}(K \mid A)^{\mathrm{zar}}$. It can also be thought of as being those valuation domains in $\operatorname{Zar}(K \mid A)$ whose maximal ideal dominates $\left(T_{1}, T_{2}\right)$ in $A$. Hence, it contains the minimal valuation overrings and is sufficient to generate the $b$ operation. We can let this collection be denoted by $Y^{\prime \prime}$ in Theorem 4.13 .

- The set $Y^{\prime \prime}$, described above, is a (proper) closed subset of $\operatorname{Zar}(K \mid A)^{\mathrm{zar}}$. Hence, it is also closed in $\operatorname{Zar}(K \mid A)^{\text {cons }}$. Moreover, any closed subset of $\operatorname{Zar}(K \mid A)^{\text {cons }}$ is compact. Hence, to obtain our set $Y^{\prime}$, we can choose any closed subset of $\operatorname{Zar}(K \mid A)^{\text {cons }}$ which contains $Y^{\prime \prime}$. Since any single point is closed in $\operatorname{Zar}(K \mid A)^{\text {cons }}$, we can let $Y^{\prime}$ be the union of $Y^{\prime \prime}$ and any other single valuation overring, for example, the localization of $A$ at a height-one prime.

- The set $Y$ should contain all overrings of its members. An obvious choice then is to let $Y$ be all of $\operatorname{Zar}(K \mid A)^{\text {cons }}$. Since this is the entire space, it is trivially closed (in $\operatorname{Zar}(K \mid A)^{\text {cons }}$ ) and generates the $b$-operation.

This then gives three different sets $Y^{\prime \prime} \subset Y^{\prime} \subset Y$ with the notation of Theorem 4.13. all associated with the same (semi)star operation.

By using Remark 2.2, we can restate Corollaries 4.10 and 4.14 as follows:

4.16. Corollary. Let $A$ be an integrally closed domain and let $K$ be its quotient field. Then the following conditions are equivalent:

(i) $A$ is a vacant domain.

(ii) Each representation of $A$ is dense in $\operatorname{Zar}(K \mid A)$, with respect to the inverse topology.

4.17. Corollary. Let $A$ be an integral domain and let $K$ be its quotient field. Let $Y$ be a subset of $\operatorname{Zar}(K \mid A)$. Then, $\left(\bigwedge_{Y}\right)_{f}=\bigwedge_{C l^{\text {inv }}(Y)}$.

\section{ACKNOWLEDGEMENT}

The authors would like to thank the referee for suggestions which substantially improved the paper.

\section{REFERENCES}

[1] Paul-Jean Cahen, Alan Loper, and Francesca Tartarone, Integer-valued polynomials and Prüfer v-multiplication domains, J. Algebra 226 (2000), 765-787. MR.1752759 (2001i:13025)

[2] Claude Chevalley and Henri Cartan, Schémas normaux; morphismes; ensembles constructibles, Séminaire Henri Cartan 8 (1955-1956), Exp. No. 7, 1-10. 
[3] David E. Dobbs, Richard Fedder, and Marco Fontana, Abstract Riemann surfaces of integral domains and spectral spaces. Ann. Mat. Pura Appl. 148 (1987), 101-115. MR.932760 (89f:14002)

[4] David E. Dobbs and Marco Fontana, Kronecker Function Rings and Abstract Riemann Surfaces, J. Algebra 99 (1986), 263-274. MR836646 (87e:14001)

[5] James Dugundji, Topology, Allyn and Bacon, Boston, 1966. MR0193606 (33:1824)

[6] Alice Fabbri, Kronecker function rings of domains and projective models, Ph.D. Thesis, Università degli Studi "Roma Tre", 2010.

[7] Carmelo A. Finocchiaro, Marco Fontana and K. Alan Loper, Ultrafilter and constructible topologies on spaces of valuation domains, Comm. Algebra (to appear).

[8] Marco Fontana, Topologically defined classes of commutative rings, Ann. Mat. Pura Appl. 123 (1980), 331-335. MR.581935 (81j:13001)

[9] Marco Fontana and James Huckaba, Localizing systems and semistar operations, in "NonNoetherian commutative ring theory", 169-197, Math. Appl., 520, Kluwer Acad. Publ., Dordrecht, 2000. MR:1858162 (2002k:13001)

[10] Marco Fontana and K. Alan Loper: Kronecker function rings: a general approach, in Ideal theoretic methods in commutative algebra (Columbia, MO, 1999), 189-205, Lecture Notes in Pure and Appl. Math., 220, Dekker, New York, 2001. MR.1836601 (2002h:13029)

[11] Marco Fontana and Alan Loper, A Krull-type theorem for the semistar integral closure of an integral domain. Commutative algebra. AJSE, Arab. J. Sci. Eng. Sect. C Theme Issues 26 (2001), no. 1, 89-95. MR1843459(2002e:13019)

[12] Marco Fontana and K. Alan Loper, Cancellation properties in ideal systems: a classification of e.a.b. semistar operations, J. Pure Appl. Algebra 213 (2009), 2095-2103. MR2533308 (2010d:13002)

[13] Marco Fontana and K. Alan Loper, The patch topology and the ultrafilter topology on the prime spectrum of a commutative ring, Comm. Algebra 36 (2008), 2917-2922. MR2440291 (2009d:13001)

[14] J. Fresnel and M. van der Put, Géométrie analytique rigide et applications, Progress in Mathematics 18, Birkhäuser, Basel, 1981. MR644799 (83g:32001)

[15] Robert Gilmer, Multiplicative ideal theory, Marcel Dekker, New York, 1972. MR.0427289 $(55: 323)$

[16] Alexander Grothendieck et Jean Dieudonné, Éléments de Géométrie Algébrique I, Springer, Berlin, 1970.

[17] Melvin Hochster, Prime ideal structure in commutative rings, Trans. Amer. Math. Soc. 142 (1969), 43-60. MR0251026(40:4257)

[18] Franz Halter-Koch, Kronecker function rings and generalized Integral closures. Comm. Algebra 31 (2003), 45-59. MR1969212 (2004e:13004)

[19] Olivier Kwegna Heubo, Kronecker function rings of transcendental field extensions, Comm. Algebra 38 (2010), 2701-2719. MR2730273 (2011k:13006)

[20] Roland Huber, Bewertungsspektrum und rigide Geometrie, Regensburger Mathematische Schriften, vol. 23, Universität Regensburg, Fachbereich Mathematik, Regensburg, 1993. MR 1255978 (95c:32036)

[21] Roland Huber and Manfred Knebusch, On valuation spectra, in "Recent advances in real algebraic geometry and quadratic forms: proceedings of the RAGSQUAD year", Berkeley, 19901991, Contemp. Math. 155, Amer. Math. Soc. Providence RI, 1994. MR1260707 (95f:13002)

[22] Franz-Viktor Kuhlmann, Places of algebraic fields in arbitrary characteristic, Advances Math. 188 (2004), 399-424. MR2087232 (2005h:12014)

[23] Bruce Olberding, Holomorphy rings of function fields, in "Multiplicative ideal theory in commutative algebra", 331-347, Springer, New York, 2006. MR2265818 (2008f:13035)

[24] Bruce Olberding, Irredundant intersections of valuation overrings of two-dimensional Noetherian domains, J. Algebra 318 (2007), 834-855. MR2371974 (2009b:13057)

[25] Bruce Olberding, Overrings of two-dimensional Noetherian domains representable by Noetherian spaces of valuation rings, J. Pure Appl. Algebra 212 (2008), 1797-1821. MR2400744 (2009c:13008)

[26] Bruce Olberding, On Matlis domains and Prüfer sections of Noetherian domains, in "Commutative Algebra and its Applications, Proceedings of the Fifth International Fez Conference on Commutative Algebra and Applications", Fez, Morocco, 2008 (Edited by M. Fontana, 
S.-E. Kabbaj, B. Olberding, and I. Swanson), Walter de Gruyter, Berlin, New York 2009, pages 321-332. MR2640313 (2011c:13039)

[27] Niels Schwartz, Compactification of varieties, Ark. Mat. 28 (1990), 333-370. MR 1084021 (92i:14022)

[28] Niels Schwartz and Marcus Tressl, Elementary properties of minimal and maximal points in Zariski spectra, J. Algebra 323 (2010), 698-728. MR.2574858 (2010k:13006)

[29] John Tate, Rigid analytic spaces, Invent. Math. 12 (1971), 257-269. MR0306196 (46:5323)

[30] Oscar Zariski, The reduction of singularities of an algebraic surface, Ann. Math. 40 (1939), 639-689. MR0000159 (1:26d)

[31] Oscar Zariski, The compactness of the Riemann manifold of an abstract field of algebraic functions, Bull. Amer. Math. Soc. 50 (1944), 683-691. MR0011573 (6:186b)

[32] Oscar Zariski and Pierre Samuel, Commutative Algebra, Volume 2, Springer Verlag, Graduate Texts in Mathematics 29, New York, 1975 (First Edition, Van Nostrand, Princeton, 1960).

Dipartimento di Matematica, Università degli Studi "Roma Tre", Largo San Leonardo Murialdo, 1, 00146 Rome, Italy

E-mail address: carmelo@mat.uniroma3.it

Dipartimento di Matematica, Università degli Studi "Roma Tre", Largo San Leonardo Murialdo, 1, 00146 Rome, Italy

E-mail address: fontana@mat.uniroma3.it

Department of Mathematics, Ohio State University, Newark, Ohio 43055

E-mail address: lopera@math.ohio-state.edu 